\title{
Correlation of Altmetric Attention Score and Citations for High-Impact General Medicine Journals: a Cross-sectional Study
}

\author{
Amr F. Barakat, $M D^{7}$, Nayef Nimri, MD², Mohamed Shokr, $M D^{3}$, Dhruv Mahtta, MD4, \\ Hend Mansoor, PharmD, MS ${ }^{5}$, Ahmad Masri, MD', and Islam Y. Elgendy, MD ${ }^{6}$
}

${ }^{1}$ Heart and Vascular Institute, University of Pittsburgh Medical Center, Pittsburgh, PA, USA; ${ }^{2}$ Department of Medicine, Cleveland Clinic Foundation, Cleveland, OH, USA; ${ }^{3}$ Division of Cardiovascular Medicine, Department of Medicine, Wayne State University, Detroit, MI, USA; ${ }^{4}$ Department of Medicine, University of Florida, Gainesville, FL, USA; ${ }^{5}$ Department of Health Services Research, Management and Policy, University of Florida, Gainesville, FL, USA; ${ }^{\circ}$ Division of Cardiovascular Medicine, Department of Medicine, University of Florida, Gainesville, FL, USA.

J Gen Intern Med 34(6):825-7

DOI: $10.1007 / \mathrm{s} 11606-019-04838-6$

(c) Society of General Internal Medicine 2019

\section{INTRODUCTION}

Altmetric Attention Score (AAS) is an emerging tool that measures the online presence of published articles. AAS represents a sum of article mentions on social media, e.g., Twitter, and news outlets (Digital Science, London, UK). AAS is increasingly used by journals as an integral article metric. While some studies have assessed the correlation between AAS and the number of citations in several disciplines and specialties, ${ }^{1-4}$ it remains unclear whether AAS correlates with article citations among high-impact general medicine journals.

\section{METHODS}

We compiled full-length original articles published in 2014 at the three highest impact factor journals: New England Journal of Medicine (NEJM), the Lancet, and Journal of the American Medical Association (JAMA) (according to Journal Citation Reports ${ }^{\circledR}$ 2017: category "General Internal Medicine"). For each article, the reported AAS was extracted (i.e., a higher score corresponds to increased online presence, with a score of $\geq 20$ generally representing a high-performing article compared to its contemporaries). Citation counts in the subsequent 3 years (i.e., 2015-2017) were retrieved by searching Web-of-Science for the corresponding article. Pairwise correlations were done using Spearman's rank correlation coefficient for non-parametric variables and Pearson's correlation coefficient for linear variables. A simple linear regression model was used to determine the relationship between log (AAS) and $\log$ (total 3-year citations). To account for the clustering effect between the journals, a linear regression with Huber-White cluster sandwich estimator of variance was performed.

Published online January 31, 2019

\section{RESULTS}

We retrieved 551 full-length original research articles: 215 (39\%), $182(33 \%)$, and $154(28 \%)$ from NEJM, JAMA, and the Lancet, respectively. The median AAS was 129 (interquartile range 74,207 ); the median number of news outlets and twitter mentions were $8(4,15)$ and $49(27$, $95)$, respectively. The median total number of citations at 3 years was $96(50,190)$. The correlation coefficient for the AAS and 3 -year citations was $0.33(P<0.001)$ (Table 1).

On simple linear regression, log (AAS) was a positive predictor of $\log$ (3-year citations) $(P<0.001)$, and the model was statistically significant $(P<0.001)$. However, the model only explained $12 \%$ of this relationship $\left(R^{2}=\right.$ 0.12) (Fig. 1a). The adjustment for article type improved $R^{2}$ to 0.16 , but did not significantly affect the overall model. By accounting for the clustering effect between journals in the regression model, $R^{2}$ remained 0.12 , but the model did not reach statistical significance $(P=0.09)$ (Fig. 1b). Accounting for the journal impact factor as a continuous variable showed a 0.03 increase in the log (total Citations) for each 1-point increase in the impact factor $(P<0.001)$.

\section{DISCUSSION}

In this cross-sectional analysis of full-length original research articles published in specific high-impact general medicine journals, we demonstrated that AAS poorly correlated with the number of citations in the subsequent 3 years. The correlation strength did not change on subgroup analyses of individual journal, study type, or the individual score components. The correlation between AAS and the total citations over 3 years was further diminished after adjusting for the clustering effect between journals.

Given the increasing interest in the use of social media to promote articles, it is important to understand the impact of new online attention metrics on more conventional article metrics, such as article citations. One study of 16 
Table 1 Descriptive Characteristics of the Included Articles and the Studied Correlations Between Altmetric Attention Score and Total Citations

\begin{tabular}{|c|c|}
\hline Variable & $N=551$ \\
\hline \multicolumn{2}{|l|}{ Journal, No. (\%) } \\
\hline NEJM & $215(39 \%)$ \\
\hline$J A M A$ & $182(33 \%)$ \\
\hline The Lancet & $154(28 \%)$ \\
\hline \multicolumn{2}{|l|}{ Article type, No. (\%) } \\
\hline Clinical trial & $291(52.8 \%)$ \\
\hline NEJM & 125 \\
\hline$J A M A$ & 82 \\
\hline The Lancet & 84 \\
\hline Observational study & $229(42.6 \%)$ \\
\hline NEJM & 89 \\
\hline$J A M A$ & 88 \\
\hline The Lancet & 52 \\
\hline Systematic reviews and meta-analysis & $31(5.6 \%)$ \\
\hline NEJM & 1 \\
\hline$J A M A$ & 12 \\
\hline Lancet & 18 \\
\hline Altmetric score, median (IQR) & $129(74,207)$ \\
\hline NEJM & $134(75,209)$ \\
\hline$J A M A$ & $124(87,194)$ \\
\hline The Lancet & $100(41,212)$ \\
\hline News outlet mentions & $8(4,15)$ \\
\hline Twitter mentions & $49(27,95)$ \\
\hline 3-year total citations, median (IQR) & $96(50,190)$ \\
\hline Citations in 2015 & $31(14,62)$ \\
\hline NEJM & $51(30,99)$ \\
\hline$J A M A$ & $16(9,35)$ \\
\hline The Lancet & $25(13,47)$ \\
\hline Citations in 2016 & $34(18,68)$ \\
\hline NEJM & $58(32,102)$ \\
\hline$J A M A$ & $21(13,42)$ \\
\hline The Lancet & $27(18,59)$ \\
\hline Citations in 2017 & $30(16,62)$ \\
\hline NEJM & $45(25,102)$ \\
\hline$J A M A$ & $19(12,36)$ \\
\hline The Lancet & $26(16,52)$ \\
\hline \multicolumn{2}{|l|}{ Spearman correlation $^{\mathrm{a}}$, correlation coefficient } \\
\hline Altmetric score and 3-year total citations & 0.33 \\
\hline Clinical trials & 0.32 \\
\hline Observational studies & 0.31 \\
\hline Systematic reviews and meta-analyses & $.009^{\mathrm{b}}$ \\
\hline General medicine articles $(n=151)$ & 0.32 \\
\hline Subspecialty articles $(n=400)$ & 0.37 \\
\hline Primary care topics $(n=368)$ & 0.29 \\
\hline Hospital-based topics $(n=183)$ & 0.39 \\
\hline Altmetric score and first-year citations ${ }^{\mathrm{c}}$ & 0.34 \\
\hline Altmetric score and second-year citations & 0.32 \\
\hline Altmetric score and third-year citations & 0.32 \\
\hline News outlet mentions and 3-year total citations & 0.29 \\
\hline Twitter mentions and 3-year total citations & 0.16 \\
\hline \multicolumn{2}{|c|}{ Altmetric score percentile ranks and 3-year total citations ${ }^{\mathrm{d}}$} \\
\hline Altmetric score in the top $50 \%$ & $0.15^{\mathrm{e}}$ \\
\hline Altmetric score in the top $25 \%$ & $0.08^{\mathrm{f}}$ \\
\hline Altmetric score in the top $10 \%$ & $0.18^{\mathrm{g}}$ \\
\hline Altmetric score in the top $1 \%$ & $0.20^{\mathrm{h}}$ \\
\hline
\end{tabular}

IQR, interquartile range; JAMA, Journal of the American Medical Association; NEJM, New England Journal of Medicine

${ }_{P}^{a}$ values for all correlations is $<.001$

${ }^{b} P$ value $=0.96$

${ }^{c}$ Citations in 2015

${ }^{d}$ Excluding 1 outlier article with an Altmetric score of 2902 and only 17 citations

${ }^{e} P$ value $=0.01$

${ }^{f_{P} \text { value }}=0.38$

${ }^{g} P$ value $=0.20$

${ }^{h} P$ value $=0.75$

selected articles in pain medicine found that measures of social media engagement did not correlate with article citations at 1 year. ${ }^{5}$ Another study examined the influence of tweets on the number of citations for articles published in the Journal of Medical Internet Research and found a correlation between number of tweets within 3 days of publication and future citations. ${ }^{6}$ A study comparing the correlation of AAS and citations in the 6 PLOS journals showed no significant correlation for PLOS Medicine. ${ }^{4}$ Our study extends beyond these findings by including the highest impact general medicine journals, which are expected to have the highest AAS for their articles due to 

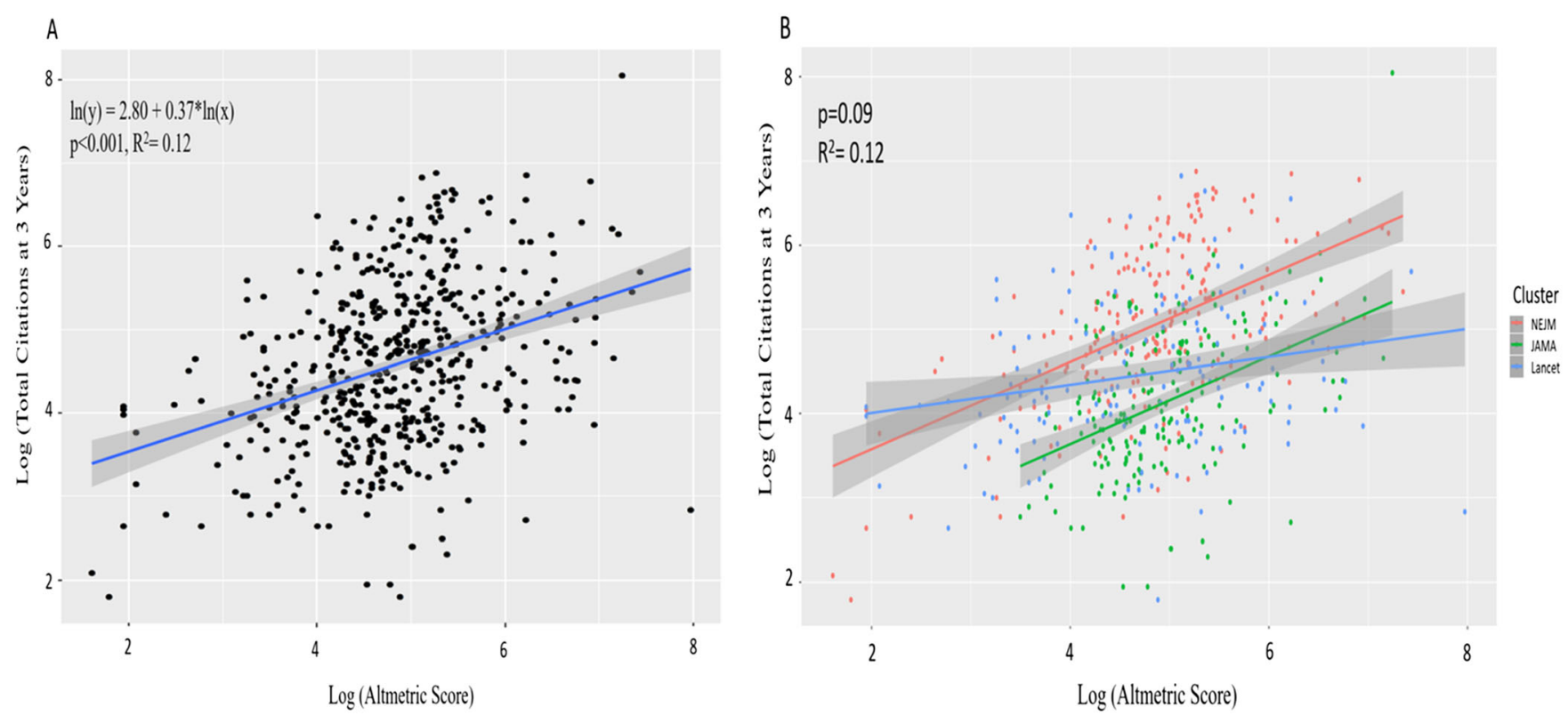

Fig. 1 a Scatter plot examining the relationship between log (Altmetric Attention Score) and log (3-year total citations), with a fitted line representing the regression model and 95\% confidence interval. b Scatter plot examining the relationship between log (Altmetric Attention Score) and $\log$ (3-year total citations) after adjusting for the clustering by journal, with fitted lines representing the regression models and 95\% confidence intervals. Red line: The New England Journal of Medicine (NEJM); green line: Journal of the American Medical Association (JAMA); green line: The Lancet.

wider audience. However, we could not comment on the effect of article accessibility (i.e., open versus closed access) on this association. It is also important to highlight that these results might be of limited generalizability to other subspecialty journals. For example, a similar analysis found that AAS moderately correlated with citations for cardiology articles, ${ }^{3}$ which might be related to the variation in social media engagement among the different specialties.

Corresponding Author: Islam Y. Elgendy, MD; Division of Cardiovascular Medicine, Department of Medicine, University of Florida, 1600 SW Archer Road, PO Box 100288, Gainesville, FL 32610, USA (e-mail: iyelgendy@gmail.com).

\section{Compliance with Ethical Standards:}

Conflict of Interest: The authors declare that they do not have a conflict of interest.
Publisher's Note: Springer Nature remains neutral with regard to jurisdictional claims in published maps and institutional affiliations.

\section{REFERENCES}

1. Thelwall $\mathbf{M}$, Haustein $\mathbf{S}$, Larivière V, Sugimoto $\mathbf{C R}$. Do altmetrics work? Twitter and ten other social web services. PLoS One. 2013;8:e64841.

2. Barbic D, Tubman M, Lam H, Barbic S. An Analysis of Altmetrics in Emergency Medicine. Acad Emerg Med. 2016;23:251-68.

3. Barakat AF, Nimri N, Shokr $\mathbf{M}$ et al. Correlation of Altmetric Attention Score With Article Citations in Cardiovascular Research. J Am Coll Cardiol. 2018;72:952-953.

4. Huang W, Wang $\mathbf{P}$, Wu $\mathbf{Q}$. A correlation comparison between Altmetric Attention Scores and citations for six PLOS journals. PLoS One. 2018; 13:e0194962.

5. Allen HG, Stanton TR, Di Pietro F, Moseley GL. Social media release increases dissemination of original articles in the clinical pain sciences. PLoS One. 2013;8:e68914.

6. Eysenbach G. Can tweets predict citations? Metrics of social impact based on Twitter and correlation with traditional metrics of scientific impact. J Med Internet Res. 2011;13:e123. 